\title{
Grazia Prontera
}

\section{Das Emigrationszentrum in Verona}

\section{Anwerbung und Vermittlung italienischer Arbeitskräfte in die Bundesrepublik Deutschland 1955-1975}

Die italienische Zuwanderung in die Bundesrepublik Deutschland nach dem Zweiten Weltkrieg entwickelte sich zum einen im Rahmen eines bilateralen Anwerbeabkommens und zum anderen im Rahmen der Römischen Verträge, die die Freizügigkeit von Arbeitskräften innerhalb der Europäischen Wirtschaftsgemeinschaft (EWG) ermöglichten. Damit bietet sich die italienische Arbeitsmigration in die Bundesrepublik Deutschland als beispielhaft an, um verschiedene Formen der Arbeitsmigration in Europa zu betrachten: auf der einen Seite die staatlich organisierte Migration, auf der anderen Seite die freie Bewegung von Arbeitskräften in der EWG.

Das bilaterale Abkommen unterzeichneten die Bundesrepublik Deutschland und die Republik Italien am 20.Dezember 1955. Es eröffnete die erste Phase der italienischen Abwanderung nach Deutschland, in der die Migration von staatlichen Institutionen reguliert und gesteuert sowie durch Emigrationszentren verwaltet wurde. Auf der Basis der bilateralen Abkommen bestimmten die Zielländer entsprechend ihren ökonomischen Bedürfnissen Umfang und Zusammensetzung der Arbeitsmigration. Emigrationszentren für die Vermittlung nach Deutschland gab es in Verona und in Neapel, wo die Deutsche Kommission in Italien (DKI) saß, die zuständig war für Anwerbung und Vermittlung von italienischen Arbeitskräften. Die zweite Phase der italienischen Auswanderung begann mit der Unterzeichnung der Römischen Verträge am 15. März 1957, mit denen die EWG ins Leben gerufen wurde. Um das Problem einer hohen Arbeitslosigkeit zu lösen, war es für Italien wichtig, dass die Verträge neben dem freien zwischenstaatlichen Verkehr von Waren und Kapital auch die Freizügigkeit der Arbeitskräfte festschrieben. ${ }^{1}$ Die Freizügigkeit trat allerdings erst nach einer Übergangsfrist am 8. November 1968 vollständig in Kraft. ${ }^{2}$

Bis 1961 wurden fast 65 Prozent (107030 von 165793) der italienischen Arbeitsmigranten in die Bundesrepublik Deutschland über die Emigrationszentren vermittelt, mit der Freizügigkeit änderte sich das: 1968 waren es nur noch 8 Prozent und 1972 gerade noch 1,3 Prozent (2092 von 154184). Der Großteil also reiste zu diesem Zeitpunkt auf eigene Initiative selbstständig nach Deutschland und wurde direkt von den Unternehmen angestellt. ${ }^{3}$

Im Mittelpunkt des vorliegenden Aufsatzes steht das Centro di Emigrazione (Emigrationszentrum) in Verona. Seine Geschichte verweist auf die Veränderung des Charakters der italienischen Zuwanderung in die Bundesrepublik Deutschland - von einer staatlich organisierten und „begleiteten“ hin zu einer unabhängigen und „unbegleiteten“ Arbeitsmigration. Als Quellen dienen zum einen die Akten des Emigrationszentrums in Verona selbst ${ }^{4}$, zum anderen die Akten der Deutschen Kommission sowie der Bundesanstalt für Arbeitsvermitt-

\footnotetext{
${ }^{1}$ Federico Romero, L'emigrazione operaia in Europa (1948-1973), in: Piero Bevilacqua/Andreina De Clementi/Emilio Franzina (Hrsg.), Storia dell'emigrazione italiana I, Rom 2001, S.397-414, hier S.403. ${ }^{2}$ Vgl. ebenda.

${ }^{3}$ Amtliche Nachrichten der Bundesanstalt für Arbeit, Beschäftigte ausländische Arbeitnehmer im Bundesgebiet nach ausgewählten Staatsangehörigkeiten 1954 bis 1973, Arbeitsstatistik 1973, Nürnberg 1974, S.46.

${ }^{4}$ Die Akten des Emigrationszentrums in Verona lagern seit dessen Schließung im Jahr 1982 im Keller der Direzione Provinciale del Lavoro (Direktion des Provinzarbeitsamts). Die für die Rekonstruktion der Geschichte des Migrationszentrums benutzten Jahresberichte waren zur Zeit meiner Forschung nicht archiviert und wurden in diesem Text als „Bestand Emigrationszentrum Verona“ (BEV) kenntlich gemacht. Ab 2010 wurden die Akten ins Staatsarchiv Verona verbracht.
} 
lung und Arbeitslosenversicherung, die im Bundesarchiv in Koblenz eingesehen werden können. Mithilfe dieser Akten lässt sich die beinahe unbekannte Geschichte des Emigrationszentrums schreiben und die Arbeit der DKI beleuchten, außerdem ermöglichen sie es, die Dimensionen und die Dynamik der staatlich organisierten Zuwanderung in die Bundesrepublik Deutschland nachzuzeichnen.

\section{Das deutsch-italienische Abkommen und die Etablierung des Emigrationszentrums in Verona}

Die Abwanderung wurde in Italien sowohl von der Politik als auch von der Wirtschaft als Ausweg aus der schweren Arbeitslosigkeit und somit als unumgänglicher Schritt zur Lösung der ökonomischen Probleme Italiens gesehen. Die Aufmerksamkeit der Regierung galt dementsprechend hauptsächlich dem "quantitativen Faktor“ der Emigration, das heißt: Die italienische Seite war daran interessiert, dass möglichst viele Arbeitslose im Ausland eine Beschäftigung fanden. ${ }^{5}$ Die Direzione generale dell'Emigrazione, die dem Außenministerium unterstellt war, schloss zwischen 1946 und 1955 insgesamt 14 bilaterale Abkommen, der deutsch-italienische Vertrag war das letzte dieser Art. 1946 hatte Italien bilaterale Abkommen mit Belgien und Frankreich unterzeichnet, ein Jahr später mit Großbritannien, der Tschechoslowakischen Republik, mit Schweden und Argentinien, 1948 mit Luxemburg, der Schweiz und den Niederlanden, 1950 mit Brasilien, 1951 mit dem Saarland und Australien, 1952 mit Ungarn. ${ }^{6}$ Für die Bundesrepublik war der Vertrag mit Italien der erste und galt als „Muster“ für die folgenden Abkommen, die im Laufe der 1960er Jahre mit Nicht-EWGLändern geschlossen wurden: 1960 mit Griechenland und Spanien, 1961 mit der Türkei, 1963 mit Marokko, 1964 mit Portugal, 1965 mit Tunesien und 1968 mit Jugoslawien. ${ }^{7}$

Über die Emigrationszentren in Mailand, Genua, Neapel, Messina und Verona gingen die ersten Migranten aus Italien in die USA, nach Kanada und Australien sowie in einer nächsten Phase in einzelne Beschäftigungsbereiche in Europa, wie die Landwirtschaft und den Wohnungsbau in Frankreich, den Bergbau in Belgien, die Bauwirtschaft und die Industrie in der Schweiz sowie in Westdeutschland. ${ }^{8}$ Die Besonderheit des Emigrationszentrums in Verona bestand darin, dass dort ausschließlich die Deutsche Kommission angesiedelt war, während in Mailand beispielsweise drei Kommissionen ständig (die belgische, die französische und die britische) und mehrere zeitweilig (die niederländische, die schwedische, die brasilianische und die südafrikanische) vertreten waren. Hinzu kam in Mailand das Zwischenstaatliche Komitee für Europäische Migration, das die Angehörigen der nach Übersee abgewanderten Arbeitskräfte betreute.

Das Emigrationszentrum in Verona bestand von 1956 bis 1982, dann wurden die Funktionen der DKI nach Rom verlagert, an den Hauptsitz der Deutschen Kommission. Zwischen 1960 und 1966, als die Nachfrage nach Arbeitskräften in Westdeutschland massiv stieg, hatte die DKI neben der Zentrale in Rom und der Zweigstelle in Verona einen weiteren

\footnotetext{
${ }^{5}$ Lorenzo Bertucelli, Politica emigratoria e politica estera: Il ruolo del Sindacato, in: Vanni Blengino/ Emilio Franzina/Adolfo Pepe (Hrsg.), La riscoperta delle Americhe. Lavoratori e sindacato nell'emigrazione italiana in America Latina 1870-1970, Mailand 1994, S.147-167, hier S.151.

${ }^{6}$ Goffredo Pesci, Politica e tecnica dell'emigrazione italiana, ad uso degli operatori tecnici e dei servizi sociali dell'emigrazione, Rom 1959, S. 193.

${ }^{7}$ Ulrich Herbert, Geschichte der Ausländerpolitik in Deutschland. Saisonarbeiter, Zwangsarbeiter, Gastarbeiter, Flüchtlinge, München 2001, S.203-208.

${ }^{8}$ Vgl. Luciano Tosi, La tutela internazionale dell'emigrazione, in: Piero Bevilacqua/Andreina De Clementi/Emilio Franzina (Hrsg.), Storia dell'emigrazione italiana II, Rom 2001, S.439-456, hier S.451456.
} 
Sitz im Emigrationszentrum in Neapel. ${ }^{9}$ Grundlage für die Einrichtung des Emigrationszentrums in Verona bildete das deutsch-italienische Anwerbeabkommen. Es umfasste sieben Abschnitte, in denen Zuständigkeiten und Modalitäten der Anwerbung und Vermittlung, der Betreuung, des Lohntransfers und der Familienzusammenführung sowie der Kosten von Auswahlverfahren und Reisen festgehalten waren. ${ }^{10}$

Obwohl die Präambel das gegenseitige Interesse an der Unterzeichnung des Abkommens und dessen symbolischen sowie wirtschaftlichen Wert für den Wiederaufbau eines gemeinsamen Europas hervorhob, stellte bereits der erste Abschnitt des Abkommens das tatsächliche Kräfteverhältnis zwischen den beiden Ländern klar. ${ }^{11}$ Es oblag ausschließlich der Bundesrepublik Deutschland, ,wenn sie einen Mangel an Arbeitskräften feststellt, den sie durch Aufnahme von Arbeitern italienischer Staatsangehörigkeit beheben will“, entsprechende Verhandlungen mit der italienischen Regierung einzuleiten ${ }^{12}$ und sowohl die Berufe als auch die Anzahl der benötigten italienischen Arbeitskräfte zu bestimmen. Und so teilte der Präsident der Bundesanstalt für Arbeitsvermittlung und Arbeitslosenversicherung (BAVAV) Ende Januar 1956 allen Präsidenten der Landesarbeitsämter mit: „Die Bemühung um die Vermittlung der inländischen Arbeitslosen dürfen durch die Aufnahme italienischer Arbeitskräfte nicht beeinträchtigt werden. “13

Verantwortlich für die Rekrutierung und Anstellung der Arbeitskräfte waren für die deutsche Seite die BAVAV und für die italienische das Ministero del Lavoro e della Previdenza Sociale (Arbeitsministerium). Die BAVAV ernannte die Mitglieder der DKI, das italienische Arbeitsministerium stellte der DKI die erforderlichen Räumlichkeiten im Emigrationszentrum zur Verfügung und garantierte die Betreuung durch die lokale Arbeitsverwaltung. Die DKI sammelte die Anforderungen der deutschen Arbeitgeber und reichte sie an das italienische Arbeitsministerium weiter. Die Anforderungen sollten präzise Daten wie „Beruf, Qualifikation und etwaige andere Wünsche des Arbeitgebers bezüglich der Arbeiter, über die Art der Beschäftigung und ihre voraussichtliche Dauer, über die Besonderheiten der vorgesehenen Arbeit, über die maßgebenden Lohn- und Arbeitsbedingungen, über die Möglichkeit der Unterkunft und der Verpflegung“ beinhalten. ${ }^{14}$ Das italienische Arbeitsministerium hatte die Aufgabe, die Rekrutierungsbedingungen und Anforderungen

\footnotetext{
${ }^{9}$ Die Akten zur begleiteten Migration nach Deutschland über das Emigrationszentrum in Neapel liegen nur unvollständig im Stadtarchiv von Neapel, daher ist eine Rekonstruktion der Geschichte dieses Migrationsstromes nicht möglich.

${ }^{10}$ Vereinbarung zwischen der Regierung der Bundesrepublik Deutschland und der Regierung der Italienischen Republik über die Anwerbung und Vermittlung von italienischen Arbeitskräften nach der Bundesrepublik Deutschland, in: Amtliche Nachrichten der Bundesanstalt für Arbeitsvermittlung und Arbeitslosenversicherung 4 (1956), Nr.2, S.52-55.

${ }^{11}$ In der Präambel heißt es: „Die Regierung der Bundesrepublik Deutschland und die Regierung der Italienischen Republik, von dem Wunsch geleitet, die Beziehungen zwischen ihren Völkern im Geiste europäischer Solidarität zu beiderseitigem Nutzen zu vertiefen und enger zu gestalten sowie die zwischen ihnen bestehenden Bande der Freundschaft zu festigen, in dem Bestreben, einen hohen Beschäftigungsstand der Arbeitskräfte zu erreichen und die Produktionsmöglichkeit voll auszunutzen, in der Uberzeugung, daß diese Bemühungen den gemeinsamen Interessen ihrer Völker dienen und ihren wirtschaftlichen und sozialen Fortschritt fördern, haben die folgende Vereinbarung über die Anwerbung und Vermittlung von italienischen Arbeitskräften nach der Bundesrepublik Deutschland geschlossen"; zitiert nach: ebenda, S.52.

12 Ebenda.

${ }^{13}$ Deutsch-italienische Vereinbarung über die Anwerbung und Vermittlung von italienischen Arbeitskräften nach der Bundesrepublik Deutschland vom 20. Dezember 1955, in: Amtliche Nachrichten der Bundesanstalt für Arbeitsvermittlung und Arbeitslosenversicherung 4 (1956), Nr.2, S.44-51, hier S.44.

14 Vereinbarung zwischen der Regierung der Bundesrepublik Deutschland und der Regierung der Italienischen Republik, S.52.
} 
an die Arbeitskräfte bekannt zu machen sowie das erste berufsbezogene und medizinische Auswahlverfahren durchzuführen. Durch die DKI erfolgte die Endauswahl. Sie stellte den italienischen Arbeitskräften, die einen Arbeitsvertrag unterschrieben, die Arbeitsgenehmigung für Deutschland aus. Die deutschen Arbeitgeber behielten sich die letzte Entscheidung über die Anstellung der vorgeschlagenen Bewerber vor. Im Falle einer negativen Entscheidung verpflichtete sich die DKI dazu, abgelehnten Bewerbern einen anderen geeigneten Arbeitsplatz anzubieten.

Die gesamte Vermittlungsprozedur und die Reise wurden bezahlt von der italienischen Regierung und von den deutschen Unternehmen, die italienische Arbeitskräfte anwarben. Die Reisekosten übernahm der italienische Staat vom Herkunftsort bis zum Brenner, für die restliche Strecke zahlte der deutsche Arbeitgeber. Die Modalitäten für die Rückreise sollten mit dem deutschen Arbeitgeber individuell vereinbart werden. Für jede italienische Arbeitskraft, die angestellt wurde, mussten die deutschen Arbeitgeber 50 DM zahlen; dieser Betrag erhöhte sich stufenweise bis auf 65 DM im Jahr $1975 .{ }^{15}$

Das Abkommen dokumentierte im Anhang Muster für die Bescheinigung über die berufliche Vorauswahl, den ärztlichen Untersuchungsbogen und den Arbeitsvertrag. Der Eignungstest sollte den Umfang der Allgemeinbildung des Bewerbers nachweisen (wenn keine schriftliche Dokumentation vorlag, musste z. B. die Rechenkompetenz getestet werden), seine berufliche Bildung und eventuell vorhandene Arbeitserfahrungen im Ausland. ${ }^{16}$ Mit dem Gesundheitstest wurde der allgemeine Gesundheitszustand und die körperliche Eignung in Bezug auf die in Deutschland aufzunehmende Tätigkeit überprüft. Jeder Bewerber musste sich einer Vorauswahl durch italienische Ärzte sowie einer von deutschen Ärzten durchgeführten Enduntersuchung unterziehen. Personen, bei denen die Ärzte „Krankheiten oder Gesundheitsstörungen, welche die Eignung für die auszuführenden Tätigkeiten erheblich einschränken oder ausschließen“, feststellten, wurden nicht rekrutiert, weil sie das Zusammenleben mit anderen Menschen hätten gefährden können oder dauerhafte medizinische Betreuung benötigten. Ausgeschlossen waren Menschen mit Lungentuberkulose, übertragbaren infektiösen oder parasitären Erkrankungen, „Leiden des Verdauungsapparates, welche sich durch Umstellung der Ernährungsweise verschlimmern können“, sowie solche mit besonderer Empfindlichkeit gegenüber Veränderungen der klimatischen Bedingungen, außerdem Bewerber mit „stark einschränkenden Störungen des Seh- und Hörvermögens, Karies oder Parodontose, sowie behandlungsbedürftigem bzw. nicht-ausreichend kaufähigem Gebiß ${ }^{17}{ }^{17}$ Nur wer beide Auswahltests bestand, konnte den Arbeitsvertrag unterschreiben, womit er den deutschen Arbeitnehmern mit gleicher Qualifikation in Bezug auf Lohn- und Vertragsbedingungen sowie Arbeitsschutzbestimmungen gleichgestellt war. Allerdings durften die deutschen Unternehmen noch die Erfüllung besonderer Kriterien einfordern - so galt zum Beispiel für Männer, die bei Volkswagen arbeiten wollten, dass sie nicht kleiner als 1,65 Meter und nicht älter als 38 Jahre sein durften. ${ }^{18}$

Anfang 1956 teilte die BAVAV zunächst Kriterien für die Anwerbung und Zeiträume für die Beschäftigung von Saisonarbeitskräften mit. Den Anforderungen entsprechend vermittelte die DKI italienische Arbeitskräfte nach Deutschland für die Landwirtschaft (vom

\footnotetext{
${ }^{15}$ Deutsch-italienische Vereinbarung, S.44; BEV, Ministero del Lavoro e della Previdenza Sociale (MLPS), Centro di Emigrazione Verona, Relazione sull'attività del Centro di Emigrazione 1975, S.31.

${ }^{16}$ Bescheinigung über die berufliche Vorauslese, in: Amtliche Nachrichten der Bundesanstalt für Arbeitsvermittlung und Arbeitslosenversicherung 4 (1956), Nr.2, S.56f.

${ }^{17}$ Grundsätze über Art und Umfang der gesundheitlichen Prüfung gemäß Artikel 7 der Vereinbarung, in: ebenda, S.62f., hier S.62.

18 BEV, MLPS, Centro di Emigrazione Verona, Relazione sull'attività del Centro di Emigrazione 1971, S.31.
} 
15. Februar bis zum 15. November), für den Gartenbau (vom 1. März bis zum 15. November) und für die Ernährungswirtschaft (vom 1. Mai bis zum 30.Oktober). Hinzu kamen Arbeitskräfte für den Hoch- und Tiefbau sowie für die Nebengewerbe der Bauwirtschaft einschließlich von Steinbrüchen und Ziegeleien (vom 1. Mai bis zum 15. November), außerdem für das Gaststätten- und Beherbergungsgewerbe (vom 1.April bis zum 30.Oktober) ${ }^{19}$ Eine besondere Aufmerksamkeit galt der möglichen Anstellung von Frauen, sie war wie folgt geregelt:

„Weibliche Arbeitskräfte können nur dann angeworben werden, wenn ihre Beschäftigung zusammen mit einem anderen männlichen Familienangehörigen möglich ist (z.B. in der Landwirtschaft), ferner allgemein im Hotel- und Gaststättengewerbe (bei Unterbringung im Hause), sowie in den Fällen, in denen die weiblichen Kräfte in Unterkünften untergebracht werden, die einer besonderen Leitung und Aufsicht unterstehen. Auf die zuletzt genannte Bedingung wird von den italienischen Stellen besonderer Wert gelegt; eine Anwerbung weiblicher Arbeitskräfte für die gewerbliche Wirtschaft dürfte daher zunächst nur in Ausnahmefällen möglich sein.“20

Die DKI begann ihre Arbeit am 6. Februar 1956 im Emigrationszentrum in Mailand, wo sie bis zum 31. Mai angesiedelt war. ${ }^{21}$ Am 1.Juni 1956 zog sie nach Verona um. Die Zahl der Mitarbeiter der DKI schwankte entsprechend dem Arbeitsanfall und erreichte 1956 insgesamt 20. 1961, auf dem Höhepunkt der italienischen Arbeitsmigration nach Deutschland, waren es 84, und 1975, als das Emigrationszentrum kaum mehr Arbeitskräfte zu vermitteln hatte, nur noch 19. Folgende vier Abteilungen des DKI gab es: Direktion, Verwaltung, Vermittlung und ärztlicher Dienst. Auch die Zahl der italienischen Mitarbeiter des Emigrationszentrums variierte entsprechend dem Umfang der Migration, allerdings blieb sie stets deutlich niedriger als die der Deutschen - 1956 handelte es sich um vier, 1961 um 20 und 1975 noch um neun Angestellte. ${ }^{22}$ Deren Aufgabe war es, entsprechend den Anforderungen der DKI und mit Unterstützung der italienischen Arbeitsämter, geeignete und willige Arbeitskräfte zu finden und der DKI vorzustellen, außerdem organisierten sie die Unterbringung und Verpflegung der Migranten in Verona sowie deren Reise nach Deutschland. In den Räumlichkeiten des Emigrationszentrums gab es zudem einen sozialen, einen religiösen und einen Finanzdienst. Die Mitarbeiter des sozialen Dienstes erklärten vor allem die Bedeutung der Bestimmung der Arbeitsverträge und boten wichtige praktische Unterstützung bei der Vervollständigung fehlender Papiere. Der Finanzdienst wurde von einer Agentur der Banca Nazionale del Lavoro übernommen, erläuterte die Geldwechsel-Operationen und stellte außerdem Bankcoupons für den zukünftigen Geldtransfer der Migranten aus. Die Angestellten des religiösen Dienstes waren für die Durchführung religiöser Veranstaltungen wie den Sonntagsgottesdienst zuständig und kümmerten sich um die geistliche Unterstützung für die Ausreisenden.

Im April 1956 traf das erste Kontingent von 1389 italienischen Arbeitskräften aus dem Emigrationszentrum in Verona in der Bundesrepublik Deutschland ein. Binnen vier Monaten wurden 3545 Arbeiter rekrutiert, von denen 2125 in der Landwirtschaft beschäftigt waren. ${ }^{23}$ Die Zahl der über das Emigrationszentrum angeworbenen Arbeiter stieg jedoch schnell an, da 1959 in der Bundesrepublik Vollbeschäftigung herrschte und die Zahl der Arbeitslosen niedriger war als die erforderliche Anzahl an Arbeitskräften. ${ }^{24}$ Das Emigrations-

\footnotetext{
${ }^{19}$ Deutsch-italienische Vereinbarung, S.44.

${ }^{20}$ Ebenda, S.51.

${ }^{21}$ BEV, MLPS, Centro di Emigrazione Milano, Relazione Anno 1956, S.83f.

${ }^{22} \mathrm{BEV}$, MLPS, Centro di Emigrazione Verona, Relazione sull'attività del Centro di Emigrazione 1975, S.3, 40 .

${ }^{23}$ BEV, MLPS, Centro di Emigrazione Milano, Relazione Anno 1956, S.84.

${ }^{24}$ Herbert, Geschichte der Ausländerpolitik in Deutschland, S. 203-208.
} 
zentrum allerdings war in den ersten vier Jahren in unzureichenden Räumlichkeiten untergebracht und hatte bloß 361 Schlafplätze, davon 20 für Frauen.$^{25}$ In einem Schreiben der DKI von 1960 heißt es dazu:

„In ,meiner' Vermittlungsabteilung sind in acht reichlich primitiven Räumen zusammen 30 Personen beschäftigt [...]. In diesen Räumen müssen täglich aber noch 300-400 Arbeitskräfte abgefertigt werden, das Wort Vermittlung möchte ich in diesem Zusammenhang nicht anwenden. Hinzu kommt, daß uns ein Karteiapparat und die ärztlichen Untersuchungen stärkstens behindern, wir könnten sonst mindestens das Doppelte an Kräften durchpressen. Arbeitskräfte stehen in großer Zahl zur Verfügung, allein aus der Provinz Lecce sind uns rd. 2000 abmarschbereite Kräfte gemeldet und wir können sie nur in kleinen Raten abrufen, da die Kapazität einfach viel zu klein ist.“26

1961 zog das Emigrationszentrum in Verona um, die neuen Räume wurden vom italienischen Arbeitsminister Fiorello Sullo und vom deutschen Arbeitsminister Theodor Blank persönlich eröffnet. Das neue Gebäude in L-Form befand sich nur 300 Meter vom Hauptbahnhof entfernt, es hatte fünf Stockwerke und 165 Räume auf einer Gesamtfläche von 2400 Quadratmetern: In einem Flügel, der parallel zur Bahnlinie verlief, waren die Büros der italienischen Behörde und der DKI, der ärztliche, der soziale sowie der Finanzdienst, der Gepäckbereich und die Duschen untergebracht. Der zweite Flügel führte zu einem Innenhof und beherbergte die sanitären Anlagen, die Aufenthaltsräume, das Café, die Räume für die religiöse Begleitung, Kantinen, Kühl-, Lager- und Abstellräume, Küchen und die Heizungsanlage. In den Kantinen konnten 500 Personen essen, insgesamt gab es acht Küchen. Ursprünglich sollte das Zentrum täglich 600 Personen beherbergen und betreuen, doch da bald bis zu 1000 Personen registriert wurden, mussten die Kapazitäten des Zentrums wachsen. ${ }^{27}$ Das beeindruckende Gebäude und die hohen Aufwendungen Italiens für die Organisation der „begleiteten“ Arbeitswanderung zeigen, welch hohen Stellenwert die Migrationspolitik als Element der politischen und wirtschaftlichen Stabilisierung Italiens in dieser Zeit hatte. Die Arbeitswanderung galt als wichtigstes Mittel zur Lösung des Problems der hohen Arbeitslosigkeit und einer damit zusammenhängenden sozialen Instabilität, und zudem wurde über den Geldtransfer der Migranten ein Teil des wirtschaftlichen Aufschwungs ermöglicht.

\section{Die „begleitete“ Arbeitswanderung 1956-1975}

Anhand der Berichte der Deutschen Kommission und des Emigrationszentrums lässt sich die „begleitete“ Arbeitswanderung bis ins Detail rekonstruieren, und aus den Berichten der Bundesanstalt für Arbeitsvermittlung und Arbeitslosenversicherung ergibt sich ein Bild davon, wie die deutschen Arbeitgeber die Beschäftigung italienischer Arbeitskräfte bewerteten.

Die DKI verfasste wöchentliche, monatliche und jährliche Tätigkeitsberichte, die sie der BAVAV zusandte. Sie boten Angaben über die Anzahl der ausgewählten Arbeitskräfte sowie Übersichten über die medizinischen Untersuchungsergebnisse, die Qualifikationen und die Zielorte der Arbeitsmigranten. Darüber hinaus wurden Transportlisten erstellt, die Daten über die deutschen Betriebe dokumentierten, die italienische Arbeitskräfte beschäftigten. Die italienische Direktion des Emigrationszentrums verfasste zudem jährliche

\footnotetext{
${ }^{25}$ BEV, MLPS, Centro di Emigrazione Verona, Relazione sull'attività del Centro di Emigrazione 1957, S.8.

${ }^{26}$ Bundesarchiv Koblenz (BArch), B 119/3055, Herrn Präsident Dr. Seifriz, Landesarbeitsamt BadenWürttemberg, Verona 19.3.1960.

${ }^{27}$ BEV, MLPS, Centro di Emigrazione Verona, Relazione sull'attività del Centro di Emigrazione 1961, S. 15-19.
} 
Papiere über die interne Organisation und über die an externe Firmen vergebenen Aufträge für Dienstleistungen (u.a. Verpflegung, Reinigung und Wachdienst) sowie über die sozialen, finanziellen und religiösen Dienste.

Zwischen 1956 und 1975 wurden 338147 Arbeitskräfte aus allen Provinzen Italiens ins Emigrationszentrum nach Verona vorgeladen. Die DKI befand 302755 von ihnen nach der medizinischen und der berufsbezogenen Auswahl als geeignet für die Arbeit in der Bundesrepublik Deutschland. ${ }^{28}$ Die Zahl der Ausgereisten Italiener ist gut dokumentiert, anders als die der Rückkehrer, da die Rückreisen nicht staatlich, sondern von deutschen Unternehmen und italienischen Arbeitern direkt organisiert wurden.

Die Entwicklung der „,begleiteten“ Arbeitsmigration war zum einen beeinflusst vom Inkrafttreten des EWG-Freizügigkeitsabkommens, namentlich von den Abkommen 15/61, 38/64 und 1612/68, durch die die „unbegleitete“, nicht von staatlicher Seite organisierte Migration an Bedeutung gewann. Zum anderen folgte der Verlauf der „begleiteten“ Arbeitsmigration der wirtschaftlichen Entwicklung in Deutschland. In den Zeiten wirtschaftlichen Wachstums lenkte die Arbeitsverwaltung den Arbeitskräftestrom aus Italien in alle Produktionsbereiche und insbesondere in die Industrie mit hohem und ganzjährigem Arbeitskräftebedarf. Vor allem kamen Hilfskräfte. In Zeiten der Rezession wurden qualifizierte den ungelernten Arbeitskräften vorgezogen. Die „begleitete“ Arbeitswanderung veränderte sich zwischen Mitte der 1950er und Mitte der 1970er Jahre nach und nach und verlor ihren Saisoncharakter, weil nicht mehr Land- und Bauwirtschaft dominierten, sondern die Industrieproduktion. Die saisonale Arbeitsmigration speiste sich hauptsächlich aus dem Veneto, Kampanien und Apulien und führte nach Niedersachsen, Baden-Württemberg und Nordrhein-Westfalen. In den 1960er Jahren reisten Arbeitskräfte aus allen Regionen Süditaliens zunehmend auch nach Bayern und Hessen.

Mit der Freizügigkeit und der daraus folgenden Abnahme des Umfangs der „begleiteten“ Arbeitsmigration veränderte sich die Zuständigkeit der DKI. Nicht mehr die Rekrutierung ungelernter Arbeitskräfte stand im Vordergrund, sondern die Vermittlung von Fachkräften und Umschülern nach Deutschland. Darüber hinaus fungierte die DKI immer stärker als Quelle der Information für die BAVAV über Darstellung und Wahrnehmung Deutschlands und seiner Arbeitswelt in der italienischen Presse und Öffentlichkeit.

In den ersten drei Jahren der „begleiteten“ Arbeitswanderung dominierte die Rekrutierung für Landwirtschaft, Baugewerbe und Bergbau. Die Anwerbungen für die Landwirtschaft und für den Bausektor erlebten in dieser Zeit eine entgegengesetzte Entwicklung: Die Zahl der landwirtschaftlichen Arbeitskräfte nahm stetig ab (1956: 5788, 1957: 3309 und 1958: 2360), während die der Beschäftigten im Baugewerbe wuchs (1956: 571, 1957 : 1474, 1958: 3972). ${ }^{29}$ Allein von 1956 auf 1957 sank die Nachfrage des landwirtschaftlichen Sektors um 42,8 Prozent. Hintergrund dafür war nach Angaben der BAVAV hauptsächlich die Forderung der italienischen Regierung nach höheren Löhnen. ${ }^{30}$

1957 begann das Emigrationszentrum in Verona mit der Rekrutierung von Bergleuten, die bis dahin am Widerstand der deutschen Bergbauindustrie gescheitert war. ${ }^{31}$ Dies bedeutete den Beginn der Arbeitswanderung mit ganzjähriger Beschäftigung. 1957/58 reisten

\footnotetext{
${ }^{28}$ Für eine detaillierte Rekonstruktion der Migrationsbewegung und ihrer Charakteristiken vgl. Grazia Prontera, Partire, tornare, restare? L'esperienza migratoria dei lavoratori italiani nella Repubblica Federale Tedesca nel secondo dopoguerra, Mailand 2009, S.82-110.

${ }^{29}$ BEV, MLPS, Centro di Emigrazione Verona, Relazione sull'attività del Centro di Emigrazione 1956, S.52; 1957, S. 19-21; 1958, S. 18-25.

${ }^{30}$ BArch, B 119/3582, Bundesanstalt für Arbeitsvermittlung und Arbeitslosenversicherung (BAVAV), Zusammenfassung der Berichte der Landesarbeitsämter über die Erfahrung bei der Anwerbung und der Beschäftigung italienischer Arbeitskräfte in der Bundesrepublik Deutschland im Jahre 1957, S.1. ${ }^{31}$ Prontera, Partire, tornare, restare?, S. 64.
} 
3238 italienische Bergleute ins Ruhrgebiet. Die Auswahl der Bergleute durch die DKI erfolgte auf der Basis eines strengen Verfahrens: Sie lehnte 1050 der 4288 Bewerber in Verona $\mathrm{ab}$, weil sie entweder für nicht gesundheitlich geeignet gehalten wurden oder weil man sie als Risikopersonen für Silikose, die typische Krankheit der Bergleute, einstufte. Während für die anderen Kategorien der Anteil der für nicht geeignet gehaltenen Arbeiter 12,2 Prozent betrug, erreichte er bei den Bergleuten 24,5 Prozent.

1957 fasste der BAVAV-Präsident die ersten Erfahrungen mit den italienischen Arbeitskräften in den verschiedenen Bundesländern wie folgt zusammen:

\begin{abstract}
„Die italienischen Arbeitskräfte haben sich im Allgemeinen bei der Arbeit gut bewährt. [...] Die italienischen Landarbeiter besaßen allerdings nur in Ausnahmefällen Spezialkenntnisse, die sie zu selbständigem Arbeiten befähigten. Mit den technischen Einrichtungen der Landwirtschaft waren sie im Allgemeinen wenig vertraut und kannten sich auch nur selten mit Pferden aus. Sie eigneten sich dagegen meist vorzüglich für Hackfruchtarbeiten und Gemüsebau. Selbst wenn es bei vielen Landarbeitern einer gewissen Einarbeitungszeit bedurfte, so war doch die Bereitschaft, eine angemessene Leistung zu erzielen, unverkennbar. Im allgemeinen waren die italienischen Arbeiter zu Überstunden bereit und oft sogar daran interessiert. "32
\end{abstract}

Der gute Wille der Arbeitskräfte entsprach jedoch nicht immer dem ihrer Arbeitgeber, wie im selben Dokument zu lesen ist, da diese in vielen Fällen erst nach gerichtlicher Anordnung bereit waren, geleistete Überstunden zu bezahlen.

Im Bericht von 1959 ist über die italienischen Arbeitskräfte in der Industrie, wo sie nun überwiegend vertreten waren, zu lesen, dass mit ihnen „noch bessere Erfahrungen gemacht wurden als in den Vorjahren. [...] Auch mit den italienischen Industriearbeitern, die nun in zunehmendem Maße als Dauerarbeitskräfte beschäftigt werden, liegen fast durchweg günstige Erfahrungen vor. “33 Diese Auffassung resultierte wohl auch aus der Tatsache, dass bei den Italienern nur wenige krankheitsbedingte Fehltage angefallen waren: „Einige Bezirke“, so der Präsident der BAVAV weiter, „berichten sogar von einer niedrigeren Erkrankungshäufigkeit gegenüber einheimischen Arbeitskräften. Es komme den Italienern offenbar sehr darauf an, einen hohen Verdienst zu erreichen“. Eine einzige Ausnahme wurde vermerkt:

„Nur ein Landesarbeitsamt berichtet, daß die Erkrankungshäufigkeit deutlich über der deutscher Arbeitskräfte liege. Dieses treffe insbesondere auf leichtere Erkrankungen zu; vor allem wären Süditaliener für leichte Erkältungskrankheiten recht anfällig und legten sich schon bei geringen Erkrankungsanzeichen ins Bett. Auch kleinste Verletzungen veranlaßten manche Italiener, sich als arbeitsunfähig zu bezeichnen. Die Betriebe vermuten, daß die Lohnfortzahlung im Krankheitsfalle mit zu einem solchen Verhalten beitrage. “34

Zusammenfassend hieß es, dass die Italiener sich „im Allgemeinen sehr rasch und ohne besondere Schwierigkeiten“ eingelebt hätten, weil viele von ihnen bereits im Jahr zuvor in der Bundesrepublik Deutschland beschäftigt gewesen waren und „die Arbeitgeber durch das notwendige Verständnis und Entgegenkommen zur schnellen Eingewöhnung beigetragen“ hätten. ${ }^{35}$ Nach Meinung der BAVAV war die Eingewöhnung in Deutschland maßgeblich davon abhängig, ob es den Italienern gelang, sprachliche, klimatische und vor allem Ernährungsprobleme zu überwinden; im Zweifelsfall konnten diese Probleme schließlich sogar zur Auflösung eines Arbeitsvertrages führen. Schon vor der Ankunft der ersten italienischen Arbeitskräfte bat die BAVAV in dem Merkblatt für Arbeitgeber, das über die Modalitäten für die Anstellung und den Aufenthalt der italienischen Arbeitskräfte infor-

\footnotetext{
32 BArch, B 119/3582, BAVAV, Zusammenfassung der Berichte der Landesarbeitsämter 1957, S.5.

${ }^{33}$ BArch, B 119/3580, BAVAV, Vermittlung italienischer Arbeitskräfte im Jahre 1959, Erfahrungsbericht, S.5.

34 Ebenda, S. 6.

${ }^{35}$ BArch, B 119/3582, BAVAV, Zusammenfassung der Berichte der Landesarbeitsämter 1957, S.4.
} 
mierte, um Verständnis für die anfänglichen Anpassungsprobleme und warb darum, auf deren Bedürfnisse einzugehen:

„Italienische Arbeitnehmer müssen sich erst an die deutschen Lebensverhältnisse gewöhnen. Hierauf wird besonders in der Anfangszeit der Beschäftigung Rücksicht zu nehmen sein. Die ungewohnte Kost kann leicht Anlaß zur Unzufriedenheit sein.“

Um solchen Situationen vorzubeugen, regte die BAVAV an, italienische Essgewohnheiten zu berücksichtigen:

„Es empfiehlt sich, daß die Speisen möglichst nach italienischen Gewohnheiten (Mehlspeisen) zubereitet werden. Die Verpflegung sollte aus Morgenkaffee mit den üblichen Beigaben, Frühstück, Mittagessen, Vesper und Abendessen bestehen. ${ }^{336}$

Regelmäßig war die Ernährung ein zentrales Thema in den Berichten der BAVAV. 1957 hieß es:

„Ernährungsschwierigkeiten traten nur in verhältnismäßig geringem Umfange auf, weil sich sowohl die Landwirtschaft als auch die gewerblichen Betriebe bemühten, den Wünschen der Italiener auf heimatliche Kost entgegenzukommen. Häufig wurde den Landarbeitern Gelegenheit gegeben, an Ruhetagen für sich selbst zu kochen. Bei größeren Gruppen hat sich in der Regel ein Italiener als Koch betätigt. ${ }^{\text {“37 }}$

Und zwei Jahre später:

„Viele Betriebe haben sich noch stärker als im Vorjahr darum bemüht, bei der Beköstigung der Italiener die Besonderheiten der italienischen Küche zu berücksichtigen und z. B. Kartoffeln durch Teigwaren zu ersetzen oder auch Spaghetti und Tomatenmark zur Selbstherstellung von Abendmahlzeiten zu verbilligten Preisen zur Verfügung zu stellen. Verschiedentlich wurden auch Italiener (meist Frauen von italienischen Arbeitskräften) zum Kochen herangezogen, um eine den italienischen Gewohnheiten entsprechende Verpflegung sicherzustellen. “38

Die Fürsorge in dieser Hinsicht ging so weit, dass 1961 eine Italienische Kommission mit Sitz in Nürnberg eingesetzt wurde, deren Aufgabe es war, „in Fragen der Anpassung der italienischen Arbeitskräfte an die klimatischen, wirtschaftlichen, sozialen und ernährungsmäßigen Verhältnisse sowie der betrieblichen Betreuung zur Verfügung zu stehen“ ${ }^{39}$ Außenstellen dieser Kommission gab es in Stuttgart, Frankfurt, Saarbrücken und Köln.

Die Berichte lassen deutlich werden, dass der gesamte Anwerbeprozess offenbar den Wünschen der BAVAV entsprechend verlief. Im ersten Jahr war die Zahl der vorzeitigen Vertragsauflösungen seitens der Italiener noch sehr hoch - bis zu 30 Prozent verließen die Bundesrepublik vor Vertragsende wieder. In den folgenden Jahren wurden jedoch immer weniger Verträge vorzeitig aufgelöst, 1958 waren es 23 Prozent, 1959 nur noch 12,5 Prozent. $^{40}$

Nur ein Problem war geblieben: Das Angebot an weiblichen Arbeitskräften lag weit unterhalb der Nachfrage. 1957 waren nur 102 Frauen über das Emigrationszentrum in Verona nach Deutschland gekommen (1,3 Prozent aller vermittelten Arbeitskräfte), die hauptsächlich in der Landwirtschaft und im Gaststättengewerbe arbeiteten. 1958 lag die Zahl mit

\footnotetext{
${ }^{36}$ Merkblatt für Arbeitgeber über die Beschäftigung italienischer Landarbeiter, in: Amtliche Nachrichten der Bundesanstalt für Arbeitsvermittlung und Arbeitslosenversicherung 4 (1956), Nr.2, S.67.

${ }^{37}$ BArch, B 119/3582, BAVAV, Zusammenfassung der Berichte der Landesarbeitsämter 1957, S.5.

${ }^{38}$ BArch, B 119/3580, BAVAV, Vermittlung italienischer Arbeitskräfte im Jahre 1959, Erfahrungsbericht, S.7.

${ }^{39}$ Entsendung einer Italienischen Kommission, in: Amtliche Nachrichten der Bundesanstalt für Arbeitsvermittlung und Arbeitslosenversicherung 9 (1961), Nr.6, S. 281.

${ }^{40}$ BArch, B 119/3580, BAVAV, Vermittlung italienischer Arbeitskräfte im Jahre 1959, Erfahrungsbericht, S. 5 .
} 
231 leicht höher (2,3 Prozent), die meisten von ihnen waren nun in der Lebensmittelindustrie beschäftigt. Weil das Anwerbeverfahren vereinfacht wurde, stieg die Zahl weiblicher Arbeitskräfte 1959 auf 576 deutlich an, dennoch verharrte ihr Anteil bei 2,3 Prozent. Der Präsident der BAVAV berichtete weiterhin: „Das Stellenangebot war zwar lebhaft, doch war es sehr schwierig, Italienerinnen für die Arbeitsaufnahme in der Bundesrepublik zu interessieren. ${ }^{41}$ Nach Meinung der BAVAV lag das am Widerstand der italienischen Konsulate gegenüber der Einstellung weiblicher Arbeitskräfte und daran, dass eine „Neigung der Italienerinnen, in Deutschland Arbeit aufzunehmen, nur dann vorhanden ist, wenn sich bereits Verwandte oder Bekannte im Bundesgebiet befinden, denen man folgen will“. ${ }^{42}$

Die Jahre 1960 und 1961 waren charakterisiert durch ein starkes Wirtschaftswachstum in der Bundesrepublik, sodass der Bedarf an unqualifizierten Arbeitskräften stieg. Eine zweite Deutsche Kommission wurde daher beim Emigrationszentrum in Neapel eingerichtet. Die „begleitete“ Arbeitswanderung betraf 93284 Arbeiter und damit 66 Prozent der gesamten italienischen Zuwanderung in die Bundesrepublik. Der Bausektor dominierte weiter mit 40 Prozent aller in Verona ausgewählten Migranten, gefolgt vom Metallsektor, der mit 23 Prozent im Vergleich zum Vorjahr stark gewachsen war. Für den Bergbau wurden 2275 Arbeiter rekrutiert. 1960 waren 55 Prozent der italienischen Arbeitskräfte ganzjährig beschäftigt, sodass ihr Anteil den der Saisonarbeitskräfte überstieg. ${ }^{43}$

1961 bildete im Emigrationszentrum von Verona das Jahr mit der umfangreichsten Vermittlungstätigkeit (62467 Arbeitskräfte). Der Bausektor führte mit 41 Prozent (25066 Arbeitskräfte) weiterhin die Liste der Beschäftigungsbereiche an, es folgte der Metallsektor mit 10958 Arbeitskräften, während nur 1396 Landarbeitskräfte verzeichnet wurden. Erneut dominierte der Anteil der ganzjährig gegenüber den saisonal Beschäftigten. ${ }^{44}$ Insgesamt kamen über die Emigrationszentren in Verona und Neapel 64,6 Prozent aller Arbeitsmigranten (107030 von 165 793) nach Deutschland - 1961 wurde das letzte Jahr, in dem die „begleitete“ Emigration der überwiegend genutzte Weg war. ${ }^{45}$

1961 trat auch die Verordnung EWG 15/61 in Kraft, in der die Freizügigkeit für die ganzjährig Beschäftigten beschlossen wurde, der Inländervorrang allerdings galt weiterhin. 1962 bereits war der Großteil der in das Bundesgebiet eingereisten Italiener von den deutschen Arbeitgebern namentlich angefordert worden. Auf diese Weise konnte das langwierige Anwerbeverfahren umgangen werden. Die Netzwerke von Verwandten und Bekannten, die bereits im Ausland beschäftigt waren, wurden zum Hauptinstrument der Rekrutierung.

Folglich sank zwischen 1962 und 1965 die „begleitete“ Arbeitswanderung stetig. 1964 trat die zweite europäische Freizügigkeitsverordnung (EWG 38/64) in Kraft, die nun auch die Saisonarbeitskräfte und Grenzpendler erfasste. Der Inländervorrang büßte erheblich an Gewicht ein, die freie Ausübung nicht-selbstständiger Tätigkeit wurde erlaubt; diese war bis dahin erst nach vier Jahren regelmäßiger Beschäftigung in einem der Mitgliedstaaten möglich gewesen. 1965 belief sich folglich der Einschaltungsgrad der DKI auf nur noch 26579 von insgesamt 204288 Fällen italienischer Arbeitswanderer, die nach Deutschland kamen. ${ }^{46}$

\footnotetext{
41 Ebenda, S.7.

42 Anwerbung und Vermittlung ausländischer Arbeitnehmer, Erfahrungsbericht 1961, in: Amtliche Nachrichten der Bundesanstalt für Arbeitsvermittlung und Arbeitslosenversicherung 4 (1962), Beilage, S.14.

${ }^{43}$ BEV, MLPS, Centro di Emigrazione Verona, Relazione sull'attività del Centro di Emigrazione 1960, S.22f.

${ }^{44}$ BEV, MLPS, Centro di Emigrazione Verona, Relazione sull'attività del Centro di Emigrazione 1961, S. 24-29.

${ }^{45}$ Amtliche Nachrichten der Bundesanstalt für Arbeit, Einschaltungsgrad der deutschen Vermittlungsstellen im Ausland 1957-1970, Arbeitsstatistik 1970, Nürnberg 1971, S. 82.

${ }^{46}$ Ebenda, S. 82.
} 
Die DKI versuchte sich neu zu profilieren:

„Die Deutsche Kommission in Italien ist damit stärker zu einer Vermittlungsstelle von Qualitätskräften geworden, die die Wirtschaft naturgemäß auf dem freien Arbeitsmarkt und unter den frei ohne Verträge einreisenden Italienern nicht findet. “47

Nur die Rekrutierung von Fachkräften bzw. von Frauen bedurfte einer speziellen Förderung. Die DKI ging dabei neue Wege: „Anregungen gegenüber der Presse, dem Radio und dem Fernsehen wurden nur noch im Sinne einer stärkeren Werbung von Fach- und Spezialkräften sowie weiblichen Arbeitskräften vorgenommen. “48 Die Rekrutierung von Frauen erreichte 1961 ihren Höhepunkt mit 2942 über die Emigrationszentren von Verona und Neapel vermittelten weiblichen Arbeitskräften - allerdings waren 8809 Frauen angefordert worden. ${ }^{49}$ Gerade wegen der nach wie vor hohen Nachfrage gab die DKI die Anwerbung von Frauen 1965 erneut als Ziel aus. Die Einstellung weiblicher Arbeitskräfte stockte dennoch weiterhin: Zwischen 1965 und 1969 machten sie nur zwischen 2 und 3,9 Prozent der Ausreisenden aus. ${ }^{50} 1965$ kamen über die Emigrationszentren 819 italienische Frauen zur Arbeit nach Deutschland, 487 aus Verona und 332 aus Neapel. Beschäftigung fanden sie vor allem in der Industrie, während ihre Zahl im Hotel- und Gaststättengewerbe sehr gering war. ${ }^{51}$

Reibungslos funktionierten demgegenüber die Maßnahmen zur beruflichen Bildung und Umschulung der nach Deutschland reisenden Arbeitskräfte, die in Kooperation mit staatlichen, religiösen und gewerkschaftlichen Institutionen durchgeführt wurden. Als erstes hatte die ANAP (Associazione nazionale addestramento professionale - Nationale Vereinigung für berufliche Bildung) Fachschulen in der Toskana (Calambrone), in der Lombardei (Mailand) und in den Marken (Piobbico) eingerichtet. Die berufliche Bildung erfolgte in erster Linie in den Bereichen Metallindustrie, Hotelgewerbe und Bausektor, wobei die in Deutschland gefragtesten Berufe Dreher, Schlosser und Fräser, Einschaler sowie Zimmerer und Kellner waren. ${ }^{52}$

Die Rezession 1966/67 in Westdeutschland beschleunigte die Veränderung in der Vermittlungsarbeit der DKI. Die Wirtschaftskrise zeigte vor allem 1967 ihre Auswirkungen. Die Nachfrage nach italienischen Arbeitskräften sank, die DKI in Neapel wurde geschlossen. Die Zahl der italienischen Arbeitskräfte in Deutschland verringerte sich um insgesamt 95400 von 1966: 362144 auf 1967: $266756 .{ }^{53}$ Die Arbeitgeber stoppten die Einstellung ungelernter Arbeitskräfte zugunsten von Facharbeitern. 1966 vermittelte das Emigrationszentrum in Verona 8391 Arbeitskräfte, 1967 nur noch 3987.54

1968/69 gab es einen erneuten wirtschaftlichen Aufschwung. Diese Jahre stellten die letzten in der Geschichte des Emigrationszentrums in Verona dar, in denen sich der Jahresdurchschnitt der Registrierungen auf über 12000 und der der Ausreisen auf 10000 Arbeits-

\footnotetext{
${ }^{47}$ BArch, B 119/3020, Deutsche Kommission in Italien, Erfahrungsbericht 1965, S.3.

${ }^{48}$ Ebenda, S.9.

49 Anwerbung und Vermittlung ausländischer Arbeitnehmer, Erfahrungsbericht 1961, S.14.

${ }^{50} 1965$ (729) 2,7\%, 1966 (520) 3,8\%, 1967 (157) 3,9\%, 1968 (212) 2,0\% und 1969 (224) 2,1\%: BArch, B 119/3013, Deutsche Kommission in Italien, Beschäftigung, Anwerbung und Vermittlung italienischer Arbeitnehmer, Erfahrungsbericht 1969, S.42.

${ }^{51}$ BArch, B 119/3020, Deutsche Kommission in Italien, Erfahrungsbericht 1965, S.45.

52 Ebenda, Anlage Nr.9.

53 Amtliche Nachrichten der Bundesanstalt für Arbeit, Beschäftigte ausländische Arbeitnehmer im Bundesgebiet nach ausgewählten Staatsangehörigkeiten 1954 bis 1973, Arbeitsstatistik 1973, Nürnberg 1974, S. 12.

${ }^{54}$ BEV, MLPS, Centro di Emigrazione Verona, Relazione sull'attività del Centro di Emigrazione 1967, S.25-29.
} 
kräfte belief. ${ }^{55}$ Der wirtschaftliche Aufschwung führte in allen Sektoren wieder zu einem Zuwachs der Zahl der Arbeitsverträge für ungelernte Kräfte, sodass diese einen Anteil von 67,4 Prozent der Vermittelten ausmachten. Jene, die 1968 über das Emigrationszentrum in Verona ausreisten, stellten jedoch nur noch 8 Prozent der 130236 Italiener dar, die in die Bundesrepublik einreisten. ${ }^{56}$ Trotz des wirtschaftlichen Aufschwungs war die Ineffizienz des Emigrationszentrums in Verona offensichtlich, insbesondere aufgrund des mangelnden Ausgleichs zwischen der Nachfrage und dem Angebot an Arbeitskräften. Meldeten die Provinzarbeitsämter im ersten Drittel eines jeden Jahres Tausende von ausreisewilligen Arbeitskräften, verfügte die DKI nur über wenige Hundert Anforderungen. Im zweiten Jahresdrittel konnten zahlreiche Vermittlungsaufträge nicht erfüllt werden, weil sich die italienischen Arbeitskräfte entweder dazu entschlossen, selbst Arbeit in Deutschland zu suchen, oder sich für andere Zielländer entschieden; oder aber sie blieben in Italien in der Hoffnung auf Saisonarbeit im Frühjahr oder Sommer. 1968 fanden 7000 bis 8000 Arbeiter in der ersten Jahreshälfte wegen des Mangels an Vermittlungsaufträgen keine Beschäftigung, in der zweiten Jahreshälfte hingegen ließen sich zu wenig ausreisebereite Arbeitskräfte finden. ${ }^{57}$

In Italien gab es jedoch nach wie vor eine hohe Arbeitslosigkeit, und in ihrem Bericht an die BAVAV wies die DKI insbesondere auf die Gruppe der jungen Facharbeitskräfte unter 21 Jahren hin, von der sie meinte, es handele sich um ein nicht zu unterschätzendes Potenzial, „befinden sich doch hierunter sehr viele jener jungen Kräfte, die trotz einer mehrjährigen beruflichen Ausbildung den in der heimischen Wirtschaft ersehnten Anschluß im Beruf nicht fanden“" ${ }^{58}$ Das Hauptinteresse der DKI richtete sich in der Folge auf die Vermittlung von jungen Facharbeitskräften:

„Durch den Besuch von über 120 und den Schriftwechsel mit über 600 Ausbildungsinstituten ist die DKI über den Ausbildungsstand und über die genaue Zahl der in den einzelnen Berufszweigen ausgebildeten Schüler besser als das zuständige Unterrichtsministerium informiert. “59

Die Vermittlung von jungen Facharbeitskräften war stetig gewachsen: 1963 stellten sie nur 4 Prozent der nach Deutschland ausgereisten Arbeitskräfte, bis 1966 wuchs dieser Anteil auf 25 Prozent und bis 1969 auf 64 Prozent an. Dieser Anteil war vor allem aufgrund des Einsatzes der DKI gestiegen, die einerseits die an der Einstellung junger Facharbeitskräfte potenziell interessierten Betriebe kontaktierte und andererseits die jungen Menschen über die technischen Schulen in Italien erreichen konnte:

„Die konzentrierte Werbung richtete sich darauf, von den Ausbildungsinstituten die Abgangslisten der ausgebildeten Schüler zu erhalten. Die in dieser Liste ausgewiesenen Schüler wurden durch die Deutsche Kommission persönlich angeschrieben; ihnen wurden Kurzinformationen übersandt, aus denen sie die sich aus einer Beschäftigung in deutschen Industriebetrieben ergebenden Verdienstmöglichkeiten sowie Einzelheiten über das Arbeitsleben und die sonstigen Lebensgewohnheiten in der Bundesrepublik Deutschland ersehen und mit ihren Eltern besprechen konnten. " 60

Die jungen Menschen stammten vor allem aus den südlichen Regionen Italiens und insbesondere aus Kampanien, Apulien und Sizilien.

Seit dem 8. November 1968 galt mit dem Inkrafttreten der Verordnung EWG 1612/68 die unbegrenzte Freizügigkeit für Arbeitskräfte innerhalb der EWG. Damit verlor die „beglei-

\footnotetext{
${ }^{55}$ BEV, MLPS, Centro di Emigrazione Verona, Relazione sull'attività del Centro di Emigrazione 1968, S.26-33.

${ }^{56}$ Amtliche Nachrichten der Bundesanstalt für Arbeit, Einschaltungsgrad der deutschen Vermittlungsstellen im Ausland 1957-1970, Arbeitsstatistik 1970, Nürnberg 1971, S. 82.

${ }^{57}$ BEV, MLPS, Centro di Emigrazione Verona, Relazione sull'attività del Centro di Emigrazione 1968, S.31.

${ }^{58}$ BArch, B 119/3018, Deutsche Kommission in Italien, Erfahrungsbericht 1968, S.3.

${ }^{59}$ BArch, B 119/3013, Deutsche Kommission in Italien, Erfahrungsbericht 1969, S.22.

${ }^{60}$ Ebenda, S.30f.
} 
tete“ zugunsten der „unbegleiteten“, „freien“ Migration an Bedeutung. Die nun mögliche direkte Einstellung von Arbeitskräften war, so die Meinung der Direktion des Emigrationszentrums, nicht nur im Interesse der deutschen Unternehmer, die sich die 65 DM Vermittlungsgebühr sparen konnten, sondern auch der Arbeitskräfte. Zum einen konnten sie den bürokratischen Aufwand umgehen, zum anderen erhöhten sich so die Chancen der ungelernten und älteren Arbeitsuchenden; denn für „Arbeiter über 45 Jahre, oft Analphabeten“, galt ein Rekrutierungsverbot. ${ }^{61}$

Nach Meinung der DKI waren die Gründe für die Rücknahme von Vermittlungsaufträgen für die „begleitete“ Emigration

„zu lange Laufzeiten, Abdeckung des Auftrages mit Kräften aus anderen Ländern oder mit frei einreisenden Italienern und zu lange Laufzeiten bei Vermittlungsaufträgen für weibliche Arbeitskräfte und bei bestimmten Facharbeiterkategorien“. ${ }^{2}$

Die DKI empfahl den deutschen Unternehmen sogar „das Ausweichen auf Kommissionen bzw. Verbindungsstellen in Ländern, in deren Bereich Frauen als ausreisebereit gemeldet und daher eher zu gewinnen sind“. ${ }^{6}$

Zwischen 1970 und 1975 sank der Anteil der im Emigrationszentrum in Verona ausgewählten Arbeitskräfte auf unter 5 Prozent der Ausreisen aus Italien nach Deutschland, hauptsächlich waren es Dauerbeschäftigte in Metallberufen. In den Jahresberichten der Leitung des Emigrationszentrums wurde das Absinken der Zahl der Ausreisen vor allem auf die Abnahme der Zahl der Vermittlungsaufträge aus Deutschland zurückgeführt, wo zunehmend Arbeitnehmer aus Nicht-EWG-Staaten beschäftigt wurden. Die Freizügigkeitsverordnung privilegierte zwar die italienischen Arbeitnehmer gegenüber Arbeitskräften aus Nicht-Mitgliedstaaten, die weiterhin an eine befristete Aufenthaltsgenehmigung und an den jeweiligen Arbeitgeber gebunden blieben. Dennoch beklagte das Emigrationszentrum von 1970 an eine Verringerung der Zahl der vermittelten Stellen:

„Der Mangel an Arbeitsangeboten hat seinen Ursprung sowohl in der wirtschaftlichen Rezession, die die Bundesrepublik 1971 betroffen hat, als auch in der dauerhaften Nichtbeachtung des Prinzips der ,Gemeinschaftspräferenz für EWG-Arbeiter" seitens der deutschen Behörden, das sich darin äußert, dass die Arbeitgeber bevorzugt Arbeitskräfte aus Nicht-EWG-Ländern (Jugoslawien, Türkei, Griechenland, Spanien, Portugal, Tunesien usw.) einstellen. [...] Viele Arbeitsangebote aus Deutschland [wurden] an die Deutschen Kommissionen in Nicht-EWG-Ländern geschickt und auch die ,unbegleiteten "Arbeiter [fanden] nicht mehr in dem Umfang Anstellungen, wie sie es erhofft hatten." ${ }^{\text {"64 }}$

Während die Aktivitäten im Emigrationszentrum in Verona stark zurückgingen, wuchsen die der Deutschen Kommissionen in Jugoslawien und in der Türkei erheblich. 1971 vermittelte die DKI nur noch 4327 Arbeiter. Die Zahl der von der Deutschen Kommission in Jugoslawien ausgewählten Arbeitskräfte betrug 73 492, in der Türkei $63777 .{ }^{65}$ Die Arbeitsmigration aus diesen Ländern blieb bis zum Anwerbestopp 1973 weitgehend unter der Kontrolle der jeweiligen Deutschen Kommission ${ }^{66}$ : 1972 wurden im Durchschnitt 66 Prozent der Arbeitnehmer aus Griechenland, Jugoslawien und der Türkei über die Emigrationszentren in die Bundesrepublik vermittelt, von den Arbeitskräften aus Portugal, Spanien,

${ }^{61}$ BEV, MLPS, Centro di Emigrazione Verona, Relazione sull'attività del Centro di Emigrazione 1969, S.28.

${ }^{62}$ BArch, B 119/3013, Deutsche Kommission in Italien, Erfahrungsbericht 1969, S.14f.

63 Ebenda, S.26f.

${ }^{64} \mathrm{BEV}$, MLPS, Centro di Emigrazione Verona, Relazione sull'attività del Centro di Emigrazione 1971, S.36f.

${ }^{65}$ Amtliche Nachrichten der Bundesanstalt für Arbeit. In das Bundesgebiet neu eingereiste ausländische Arbeitnehmer nach der Staatsangehörigkeit 1964-1973, Arbeitsstatistik 1973, Nürnberg 1974, S.46.

${ }^{66}$ Der Anwerbestopp von 1973 traf nicht die EWG-Arbeitskräfte und somit auch nicht die Italiener. 
Marokko und Tunesien waren es durchschnittlich 80 Prozent. Das Emigrationszentrum in Verona vermittelte gerade einmal noch 1,3 Prozent der italienischen Arbeitskräfte. ${ }^{67}$

Wegen der drastischen Abnahme der „begleiteten“ Migration entschied sich die DKI zu einer teilweisen Neuausrichtung ihrer Arbeit - zwar blieb sie weiterhin aktiv in der Vermittlung und Ausbildung, bot sich aber zugleich an als Informationsdienst für die BAVAV, um über die „Öffentliche Meinung zur Anwerbung von italienischen Arbeitnehmern für die Bundesrepublik Deutschland“ zu berichten, im Jahr 1969 hieß es beispielsweise:

„Der Deutschen Kommission sind im Berichtsjahr keine negativen Stellungnahmen - etwa in Presse oder Rundfunk - über eine Anwerbung nach Deutschland bekanntgeworden. [...] Wenn auch über die Emigration selbst kaum negative Stimmen erhoben werden - sie wird zwar als ehrenrühriges aber nicht zu umgehendes Übel hingenommen -, so wird um so mehr über die Probleme der Emigranten im Ausland berichtet. Hauptpunkte der Berichterstattung sind die fehlenden Möglichkeiten der Berufsausbildung und die fehlenden Grundschulen für italienische Kinder in Deutschland sowie Wohnungsfragen."68

In der Folge der internationalen Wirtschaftskrise Ende 1973, die in erheblichem Maße die Automobilindustrie traf, stieg in Deutschland die Arbeitslosenzahl, sodass die Unternehmen die Nachfrage nach Arbeitskräften reduzierten und auf Fachkräfte beschränkten, die zeitweise und je nach Bedarf beschäftigt werden sollten. 1974 kamen mit 1457 Arbeitskräften 2000 weniger über das Emigrationszentrum in Verona in die Bundesrepublik als im Jahr zuvor, 1975 waren es nur noch $229 .{ }^{69}$ Das Emigrationszentrum blieb dennoch bis 1982 aktiv und erlebte 1976 mit 1034 und 1979 mit 1176 Ausreisen nach Deutschland noch zwei Höhepunkte - allerdings auf niedrigem Niveau. Mit der Schließung des Emigrationszentrums übernahm eine Kontakt- und Informationsstelle in Rom, die seit 1969 für die Pflege der Beziehungen zu den italienischen Institutionen zuständig war, die Funktionen der Deutschen Kommission. Das gesamte Aktenmaterial des Emigrationszentrums blieb im Keller des Arbeitsamts in Verona, verpackt in Pappkartons mit der Aufschrift „Archivio Morto“ - Totes Archiv.

\footnotetext{
${ }^{67} 67,3 \%$ Griechen (16602 von 24666), 63,3\% Jugoslawen (47815 von 75501), 64,8\% Türken (62394 von 96210), 87,5\% Portugiesen (14420 von 16476), 78,3\% Spanier (22463 von 28657), 75,7 \% Marokkaner (3034 von 4003), 78,8 \% Tunesier (1 517 von 1923), vgl. Amtliche Nachrichten der Bundesanstalt für Arbeit. In das Bundesgebiet neu eingereiste ausländische Arbeitnehmer nach der Staatsangehörigkeit 1964-1973, Arbeitsstatistik 1973, Nürnberg 1974, S. 46.

${ }^{68}$ BArch, B 119/3013, Deutsche Kommission in Italien, Erfahrungsbericht 1969, S.5f.

${ }^{69}$ BEV, MLPS, Centro di Emigrazione Verona, Relazione sull'attività del Centro di Emigrazione 1975, S. 1
} 